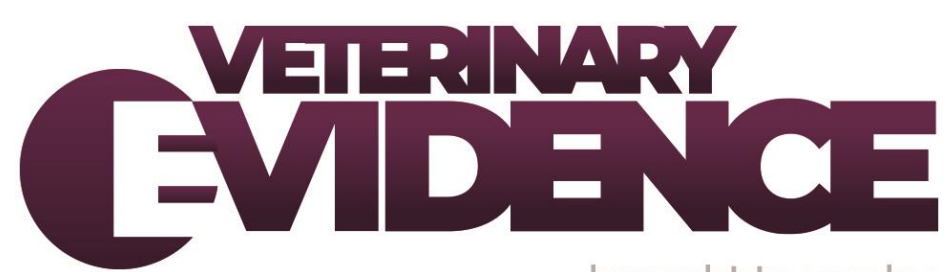

\title{
The prevalence of positive urine cultures in 100 dogs with an inactive urine sediment
}

Rachel Liebelt DVM, MRCVS ${ }^{*}$

Armi Pigott DVM, DACVECC ${ }^{2}$

\footnotetext{
${ }^{1}$ Vets Now Hospital Manchester, 98 Bury Old Rd, Whitefield, Manchester, M45 6 TQ

${ }^{2}$ Lakeshore Veterinary Specialists, 2100 W. Silver Spring Drive, Glendale, WI 53209

*Corresponding Author (rlliebelt@gmail.com)
}

ISSN: 2396-9776

Published: 19 Dec 2019

in: Vol 4, Issue 4

DOI: $10.18849 /$ VE.V4I4.273

Reviewed by: Conor O'Halloran (BVSc, MSc, MRCVS) and Mary

Thompson (BVSc(Hons), PhD, DACVIM, MANZCV) 
Objective: The purpose of this study was to determine the prevalence of positive quantitative urine cultures in dogs with an inactive urine sediment.

Background: A urinalysis is a useful screening tool for the evaluation of evidence of bacterial cystitis and a quantitative urine culture is used for definitive diagnosis.

Evidentiary value: A retrospective chart review from June 2012 to December 2017 at three private practice emergency and specialty referral hospitals examined urine samples obtained from 100 client-owned dogs.

Methods: The signalment and clinicopathologic data was recorded for all canine patients that had urine samples obtained by cystocentesis that had an inactive sediment exam on urinalysis and subsequent quantitative urine cultures were performed.

Results: The prevalence of positive quantitative urine cultures in all dogs with an inactive urine sediment at the aforementioned institutions was $6 \%$ (6/100). Escherichia coli was the most commonly isolated bacteria.

Conclusion: Given the low prevalence of positive quantitative urine cultures in dogs with an inactive urine sediment and current guideline recommendations for management of subclinical bacteriuria, we do not recommend urine cultures for dogs without lower urinary tract signs. Further prospective study of patient subgroups, as well as controlled studies evaluating urine sample handling techniques using methods available to private practice practitioners are sorely needed.

Application: Emergency and general practicing veterinarians should consider a quantitative urine culture for dogs with lower urinary tract signs, even with an inactive sediment examination, and on a case-by-case basis for dogs with pertinent systemic diseases or known risk factors for bacterial cystitis.

\section{INTRODUCTION}

Urinalysis and quantitative urine culture (QUC) are common diagnostics performed in a wide population of both human and veterinary patients. A urinalysis is a useful screening tool in the evaluation for supporting evidence of bacterial cystitis (Weese et al., 2019), however urine culture is the gold standard to diagnose or rule out bacteriuria (Ettinger, 2017). A QUC should be performed in cases of known or suspected bacterial cystitis or pyelonephritis to determine the infectious agent and guide antimicrobial usage (Weese et al., 2019; Ettinger, 2017). Urinalysis and QUC are also commonly performed as part of the routine diagnostic approach to determining sources of fever, causes for diabetic decompensation and ketosis, and as part of routine laboratory evaluation of the chronic kidney disease (CKD) or acute kidney injury patient (Ettinger, 2017).

There are occasions when a positive QUC is obtained from urine that has no evidence of bacteria or inflammation on urinalysis. This scenario may be due to the limitations of light microscopy in detecting low numbers of bacteria compared to QUC (Ettinger, 2017). The literature states that $10,000 \mathrm{rods} / \mathrm{mL}$ to 100,000 $\mathrm{cocci} / \mathrm{mL}$ of urine is required to consistently find bacteria on sediment evaluation, whereas a QUC is much more sensitive for detecting bacteria present in smaller numbers (Ettinger, 2017). 
A QUC is a relatively expensive test in comparison to the cost of a urinalysis, and it would be useful to know how often the QUC is positive when patients have no evidence of bacteria or inflammation on urine sediment examination. The authors hypothesised that positive QUCs occur in less than $10 \%$ of patients with an inactive urine sediment. The goal of this paper was to determine the prevalence of positive QUCS associated with inactive urine sediments in all patients who had a QUC performed at a private practice emergency and specialty referral hospital, which to the authors' knowledge, has not previously been reported. The secondary aim of this paper was to describe the QUC-positive patients.

METHODS \& MATERIALS

Results of all QUCs performed between June $1^{\text {st }} 2012$ to December $31^{\text {st }} 2017$ at all three locations of the authors' institutions were identified; 2,095 QUCs in total. The corresponding urinalysis for each QUC was then evaluated. An inactive sediment was defined as urine with colour descriptor recorded as yellow or colourless; turbidity descriptor recorded as clear; and the absence of epithelial cells, bacteria and pyuria (defined as greater than five white blood cells per high powered field) on urine sediment examination.

Dogs having urine collection by cystocentesis, urinalysis with inactive urine sediment, and QUC, submitted on the same day that the urine for urinalysis was collected, were included in the study. Dogs were excluded from the study if they had urinalysis and QUC performed on separate days or at separate visits, did not have a urinalysis performed, had urine collected by a method other than cystocentesis or method not recorded, had urinalysis that did not meet the defined parameters for inactive sediment examination, or had a medical record that was otherwise insufficiently complete for data extraction, as depicted in Figure 1.

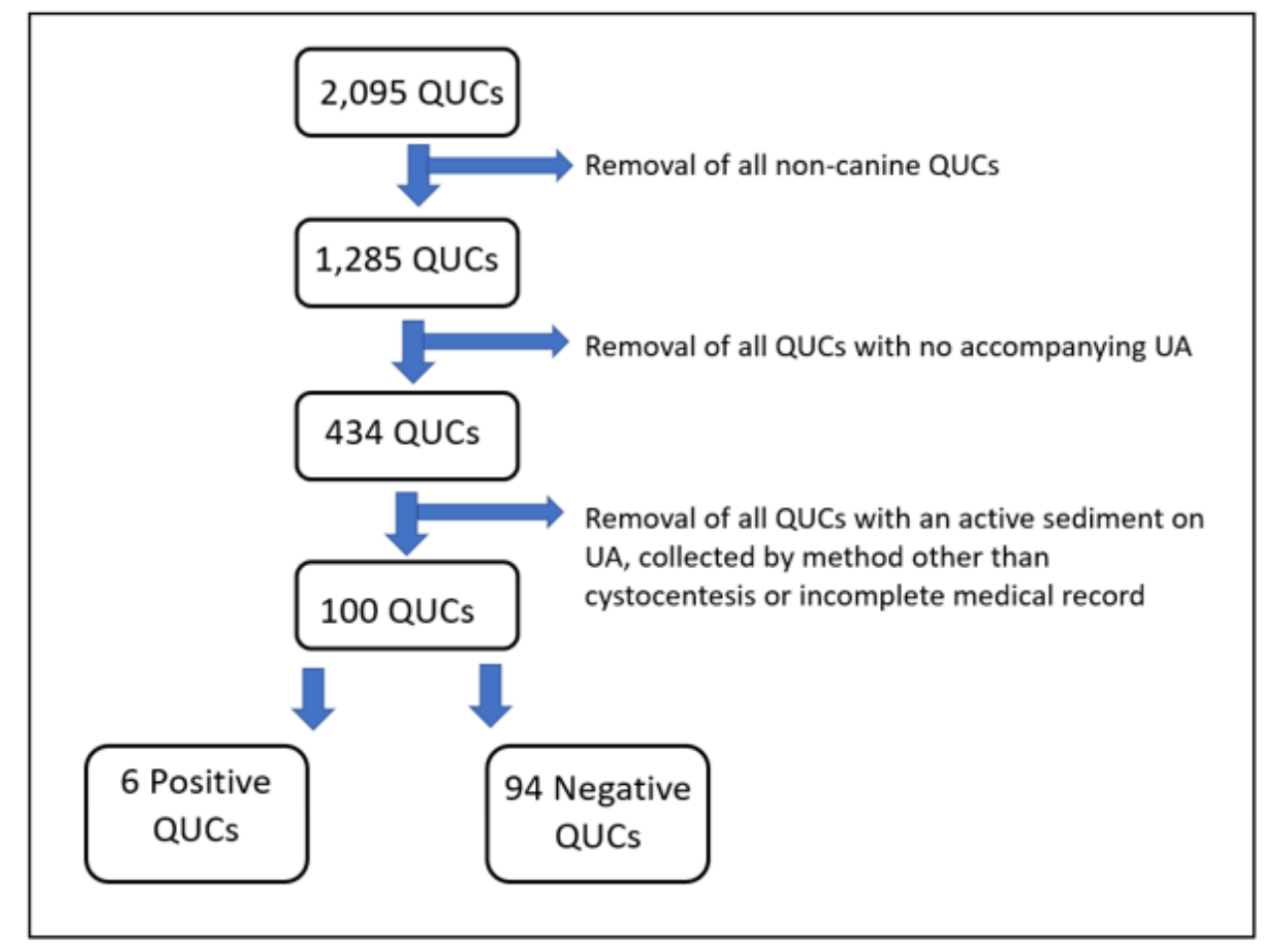

Figure 1: Flow chart depicting the exclusion process of the quantitative urine cultures (QUCs) in this study. $\mathrm{UA}=$ urinalysis 
For dogs meeting the inclusion criteria the following data were extracted from the medical record:

- Age

- Sex and reproductive status (male vs female vs spay vs neuter)

- Species

- Breed

- Urine culture result (positive vs negative)

- Urine specific gravity (USG)

- Laboratory performing the urinalysis (in-house vs commercial laboratory ${ }^{a}$ )

- Estimated time from collection of urine to culture plating (within 24 hours vs less than 40 hours vs greater than 40 hours)

- Presence of crystalluria

- Microscopic heamaturia (fewer than five red blood cells per high powered field vs greater than five red blood cells per high powered field)

- Presence or absence of lower urinary tract signs (LUTS)

- If the visit was a recheck examination following treatment for bacterial cystitis

- The presenting complaint/clinical signs of each patient

- Comorbidities that might result in decreased white blood cells in the urine despite active infection (hypothyroidism, chronic kidney disease (CKD), hyperadrenocorticism (HAC), diabetes mellitus (DM), prostatitis, urolithiasis, neurogenic bladder, pregnancy, chemotherapy)

- Recent steroid administration

- Recent antimicrobial administration

A culture was considered positive if any growth was detected, and the bacterial species and quantitative bacterial counts were recorded.

Additionally, select therapeutic data (antimicrobial prescribed, duration of antimicrobial prescription) and response to therapy (resolution of LUTS vs persistence of LUTS vs lost to follow-up) was obtained for dogs that presented for LUTS, had an inactive urine sediment and had a negative QUC. The records of dogs that presented with and without LUTS, had an inactive urine sediment and a positive QUC were also reviewed in more detail.

During the study period the urine sediment was evaluated by either in-house technical staff trained in reading urinalysis or an outside commercial laboratorya. All QUCs were performed by the same commercial laboratory ${ }^{a}$. Routine handling of urine samples after collection at the authors' institutions is as follows: immediately following collection the urine is transferred to preservative-free sample tubes $(6 \mathrm{~mL}$ max volume; provided by the commercial laboratory ${ }^{\mathrm{a}}$ ), and a patient identification label is applied to the tubes. The total volume collected is not standardised. The tubes of urine are subsequently stored in a refrigerator at $1.1^{\circ}-2.2^{\circ} \mathrm{C}$ $\left(34^{\circ}-36^{\circ} \mathrm{F}\right)$ until the sample is collected by a courier provided by the commercial laboratory ${ }^{\mathrm{a}}$. Samples are transported to the laboratory in a cooler on ice. The QUCs at the commercial laboratory are plated on a Blood Ager/MacConkey Agar Biplates (Hardy diagnostics, CA).

If the urinalysis was performed in-house it was done immediately following collection. The in-house urinalysis is performed as follows: USG is determined using a commercially available refractometer. Protein, $\mathrm{pH}$, blood, ketones, glucose, and bilirubin are evaluated using a commercially available colourimetric urine dipstick test according to manufacturer's instructions. In instances where the USG was greater than the refractometers reference range the result was recorded in the medical record as $>1.0 \mathrm{xx}$ (1.0xx being the highest mark on the 
refractometer in use at the time). When these data points were extracted from the record they were recorded as $1.0 x x+0.001$. For example; $>1.060$ was recorded as 1.061 .

Following dipstick testing, a urine sediment unstained wet mount is evaluated microscopically. This sample is prepared as follows: $1 \mathrm{~mL}$ of urine is centrifuged for 45 seconds at 9800 revolutions per minute $(3900 \times \mathrm{g})$ in a commercial centrifuge. The supernatant is decanted and sediment resuspended in the remaining fluid by gentle agitation. One drop is placed on a glass slide and covered with a cover slip for microscopic evaluation using the $10 x$ and $40 x$ objective. A minimum of 10 fields are evaluated to determine the average number of cells, casts and organisms per field.

Statistical Methods - Data were analysed by means of descriptive and inferential methods using a commercial spreadsheet software programme ${ }^{b}$. Data were tabulated for descriptive purposes. Data distribution was tested for normality using the Shapiro-Wilk test (Vetter, 2017). Comparison between groups was performed by means of Mann-Whitney tests for continuous variables, and Fisher's Exact Tests for categorical data (Neely et al., 2003). Values of $P<0.05$ were considered significant.

RESULTS

There were 100 canine QUCs with an accompanying inactive urine sediment examination. The overall prevalence of positive QUCs in this population of dogs with inactive urine sediment was 6\% (6/100) when all cases were evaluated. When the 15 QUCs that were performed specifically as a recheck examination following treatment for a recent diagnosis of bacterial cystitis were excluded, the prevalence of positive QUCs with inactive sediments was 7\% (6/85). A summary of the data obtained can be viewed in Table 1. 


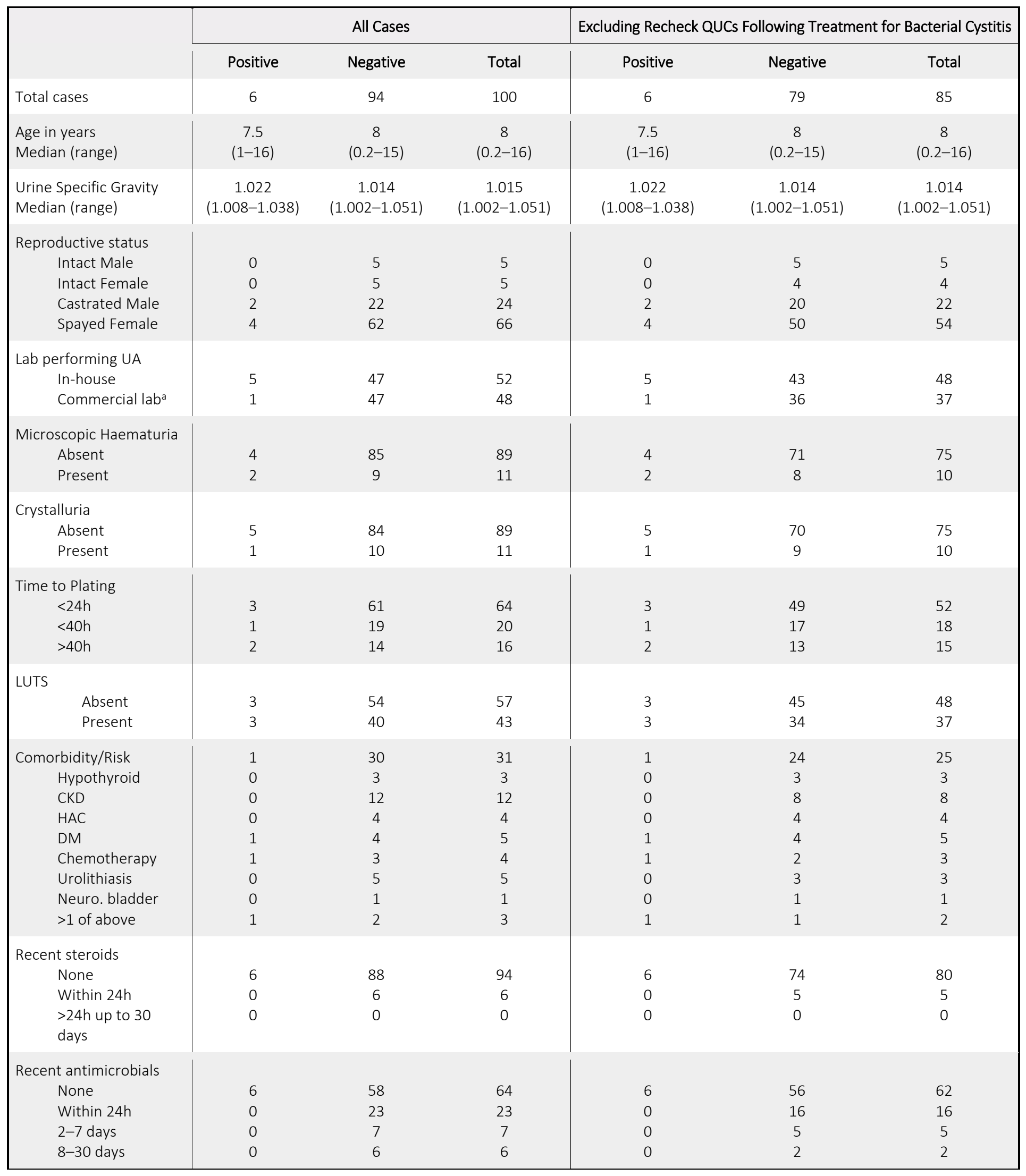

Table 1: Summary of the number of patients with inactive urine sediments in various categories.

QUC = quantitative urine culture 
$\mathrm{UA}=$ urinalysis

LUTS = lower urinary tract signs

$\mathrm{CKD}=$ chronic kidney disease

$\mathrm{HAC}=$ hyperadrenocorticism

$\mathrm{DM}=$ diabetes mellitus

Neuro. bladder $=$ neurogenic bladder

The breed distribution was as follows: $27 \%$ mixed breed, 13\% Labrador Retriever, $4 \%$ Golden Retriever, $4 \%$ German Shepherd, 3\% Corgi, 3\% Shih Tzu, and all other breeds accounted for $2 \%$ or less each. The following primary presenting complaints and/or clinical signs were retrieved from review of the medical record in the following distribution: $33 \%$ with LUTS, $12 \%$ with aezotemia, 10\% with fever, $9 \%$ with gastrointestinal tract signs, $5 \%$ with neurological signs, $4 \%$ with lethargy, $4 \%$ with dermatological concerns, $3 \%$ with anorexia, $3 \%$ for endocrine evaluation, and $2 \%$ for each of the following categories: hepatopathy, behavioural, polyuria/polydipsia, respiratory tract, orthopaedic, reproductive and weight gain. Regardless of the primary presenting complaints and/or clinical signs further review of the patient's history indicated that $44 \%$ of all dogs had LUTS described in their medical records. Fourteen variables were compared between the positive and negative QUC cases with no statistically significant differences identified. The analysis was repeated when the patients presenting for recheck following therapy for bacterial cystitis were excluded with the same (no difference) outcome. Variables and results are shown in Table 2. Additionally, there was no difference between the sediment samples based on where the urinalysis was performed (in-house vs a commercial laboratory ${ }^{a}$ ) including rate of positive culture $(P=0.207)$.

\begin{tabular}{|l|c|c|}
\hline Variable & All Cases & $\begin{array}{c}\text { Excluding Recheck QUCS } \\
\text { Following Treatment for Bacterial } \\
\text { Cystitis }\end{array}$ \\
\hline Age & $p$ & $p$ \\
\hline Breed & $>0.999$ & 0.998 \\
\hline Urine Specific Gravity & 0.174 & 0.14 \\
\hline Reproductive Status & 0.886 & 0.831 \\
\hline Microscopic Hematuria & 0.833 & 0.842 \\
\hline Crystalluria & 0.13 & 0.145 \\
\hline Time to Plating & 0.513 & 0.891 \\
\hline LUTS & 0.264 & 0.445 \\
\hline Comorbidity & 0.411 & $>0.401$ \\
\hline Recent Steroids & 0.431 & 0.668 \\
\hline Antimicrobials within 24 hours & $>0.999$ & $>0.999$ \\
\hline Antimicrobials within 7 days & 0.334 & 0.336 \\
\hline Antimicrobials within 30 days & 0.072 & 0.33 \\
\hline Presenting signs & 0.085 & 0.184 \\
\hline
\end{tabular}

Table 2: Variable comparison between groups by means of Mann-Whitney (continuous) and Fisher's Exact Test (categorical). Values of $\mathrm{P}<0.05$ significant.

QUC = quantitative urine culture 
The most commonly isolated bacteria species was $E$. coli, accounting for half of the bacterial species grown $(3 / 6 ; 50 \%)$. The results of the six positive QUCs and the accompanying signalment and clinicopathologic data are displayed in Table 3.

\begin{tabular}{|c|c|c|c|c|c|c|}
\hline & Dog 1 & Dog 2 & Dog 3 & Dog 4 & Dog 5 & Dog 6 \\
\hline Age in years & 3 & 9 & 1 & 11 & 16 & 6 \\
\hline Urine Specific Gravity & 1.008 & 1.015 & 1.038 & 1.028 & 1.012 & 1.033 \\
\hline Reproductive Status & $\begin{array}{l}\text { Spayed } \\
\text { Female }\end{array}$ & $\begin{array}{l}\text { Spayed } \\
\text { Female }\end{array}$ & $\begin{array}{l}\text { Spayed } \\
\text { Female }\end{array}$ & $\begin{array}{l}\text { Neutered } \\
\text { Male }\end{array}$ & $\begin{array}{l}\text { Spayed } \\
\text { Female }\end{array}$ & $\begin{array}{l}\text { Neutered } \\
\text { Male }\end{array}$ \\
\hline Lab Performing UA & In-House & In- House & $\begin{array}{c}\text { Commercial } \\
\operatorname{lab}^{\mathrm{a}}\end{array}$ & In-House & In-House & In-House \\
\hline Organism Cultured & $\begin{array}{c}\text { Group D } \\
\text { Streptococci } \\
\text { Non-enterococcus }\end{array}$ & E. coli & $\begin{array}{l}\text { Proteus } \\
\text { mirabilis }\end{array}$ & E. coli & E. coli & $\begin{array}{c}\text { Enterrococcus } \\
\text { spp. }\end{array}$ \\
\hline Growth (CFU/mL) & $>100,000$ & $>100,000$ & $\begin{array}{c}10,000- \\
50,000\end{array}$ & 1,000 & $\begin{array}{c}10,000- \\
50,000\end{array}$ & $10,000-50,000$ \\
\hline Microscopic Haematuria & No & Yes & No & Yes & No & No \\
\hline Crystalluria & No & No & No & Yes & No & No \\
\hline Time to Plating & $>40 \mathrm{~h}$ & $>40 \mathrm{~h}$ & $<24 h$ & $<24 h$ & $<40 \mathrm{~h}$ & $<40 h$ \\
\hline LUTS & Yes & Yes & Yes & No & No & No \\
\hline Comorbidity/Risk & None & None & None & None & None & $\begin{array}{c}\text { DM } \\
\text { Chemotherapy }\end{array}$ \\
\hline Recent Steroids & No & No & No & No & No & No \\
\hline Recent Antimicrobials & No & No & No & No & No & No \\
\hline
\end{tabular}

Table 3: Summary features of the six dogs with positive QUCs with quantification of bacterial growth reported as colony forming units (CFU/mL).

$\mathrm{UA}=$ urinalysis

LUTS = lower urinary tract signs

$\mathrm{DM}=$ diabetes mellitus

Of the six dogs that had an inactive urine sediment examination and a positive QUC, three presented on an emergency basis for evaluation of LUTS (dogs 1-3). They had unremarkable histories and physical examinations otherwise. No significant work up was performed beyond a UA and QUC. All three dogs were prescribed amoxicillin/clavulanic acid (Clavamox ${ }^{\circledR}$, Zoetis, USA) by mouth (po) every (q) 12 (h) hours, at dosages ranging from $14.6-24 \mathrm{mg} / \mathrm{kg}$ for 7 to 10 days and their LUTS were reported to have resolved.

The three dogs who had an inactive urine sediment examination and a positive QUC that did not present with LUTS represented more complex cases. Dog 4 presented as an emergency for non-specific lethargy and discomfort, difficulty ambulating and a fever of $40.7^{\circ} \mathrm{C}\left(105.3^{\circ} \mathrm{F}\right)$. Initial bloodwork revealed a mild normocytic, normochromic non-regenerate anaemia (haematocrit of $28.9 \%$; reference $37.0-55.9 \%$ ), mild elevation in creatinine (141 mmol/L: reference $35.4-123.8 \mathrm{mmol} / \mathrm{L} ; 1.6 \mathrm{mg} / \mathrm{dL}$ : reference $0.4-1.4 \mathrm{mg} / \mathrm{dL}$ ), mild increase in aspartate aminotransferase $(230 \mathrm{U} / \mathrm{L}$ : reference $0-60 \mathrm{U} / \mathrm{L})$ and mild hypoglycaemia $(3.9 \mathrm{mmol} / \mathrm{L}$ : reference $4.2-6.9 \mathrm{mmol} / \mathrm{L} ; 71 \mathrm{mg} / \mathrm{dL}$ : reference $75-125 \mathrm{mg} / \mathrm{dL}$ ). An abdominal ultrasound revealed mild pyelectasia of the left renal pelvis $(2.8 \mathrm{~mm})$ with mild dilation of proximal ureter. Scant pericardial effusion was also documented. Ultimately dog 4 was euthanised before a definitive diagnosis was achieved due to failure to respond to symptomatic and supportive care provided with intravenous balanced crystalloid therapy, various opioid analgesics and ampicillin sulbactam (Unasyn, Pfizer) at $21.8 \mathrm{mg} / \mathrm{kg} \mathrm{q} 8 \mathrm{~h}$. Dog 5 presented as an 
emergency for acute haematochezia and chronic decreased appetite with weight loss. Initial bloodwork revealed mild elevation in creatinine $(175 \mathrm{mmol} / \mathrm{L}$ : reference $35.4-123.8 \mathrm{mmol} / \mathrm{L} ; 2.3 \mathrm{mg} / \mathrm{dL}$ : reference $0.4-$ $1.4 \mathrm{mg} / \mathrm{dL}$ ), and blood urea nitrogen $(23.3 \mathrm{mmol} / \mathrm{L}$ : reference $3.2-10.4 \mathrm{mmol} / \mathrm{L} ; 65.4 \mathrm{mg} / \mathrm{dL}$ : reference $9.0-$ $29.0 \mathrm{mg} / \mathrm{dL}$ ). An abdominal ultrasound revealed bilateral renal mineralisation and a right-sided renal cyst. Hypertension was also documented (232 mmHg as measured by Doppler). Dog 5 was diagnosed with CKD and was subsequently discharged with instructions to administer subcutaneous fluids $(40 \mathrm{ml} / \mathrm{kg}$ q $24 \mathrm{~h}$ ), amoxicillin/clavulanic acid at $12.5 \mathrm{mg} / \mathrm{kg}$ po $\mathrm{q} 12 \mathrm{~h}$ and an angiotensin converting enzyme inhibitor (generic Benazepril Hydrochloride, Teva) at $0.5 \mathrm{mg} / \mathrm{kg}$ po q $12 \mathrm{~h}$ with chronic management via the Internal Medicine Service. Dog 6 had a previous incompletely excised nasal grade III mast cell tumour for which he was actively receiving lomustine (Gleostine ${ }^{\circledR}$, Amatheon) at $2.1 \mathrm{mg} / \mathrm{kg}$ po q 3-4 weeks. He had also developed DM during the course of his chemotherapy and was poorly controlled. He presented as an emergency for vomiting and diarrhoea and was subsequently diagnosed with diabetic ketoacidosis and pancreatitis. He was treated in routine fashion for his diabetic ketoacidosis. His treatment included a course of amoxicillin/clavulanic acid at $21.2 \mathrm{mg} / \mathrm{kg}$ po $\mathrm{q} 12 \mathrm{~h}$ and he continued to work with the Internal Medicine and Oncology services for his DM and mast cell tumours, respectively.

\section{DISCUSSION}

This population of canine patients evaluated at private practice emergency and specialty referral hospitals was determined to have a $6 \%$ prevalence of positive QUC with an inactive urine sediment. The majority of the bacterial isolates were cultured in quantities $>10,000 \mathrm{CFU} / \mathrm{mL}$, with a single isolate at $1,000 \mathrm{CFU} / \mathrm{mL}$. Since $\geq 100 \mathrm{CFU} / \mathrm{mL}$ is considered clinically significant in both canine and feline urine obtained by cystocentesis (Ettinger, 2017; Comer \& Ling, 1981) contamination, while still possible, is not considered likely to be a cause for any of the positive cultures in this report.

Previous studies have reported $E$. coli as the bacterial species most commonly isolated from the urinary tract (Comer \& Ling, 1981; Hallet al., 2013). Older and female dogs (both intact and spayed) are more likely to have positive urine cultures (Comer \& Ling, 1981). Four of the six positive QUCs in this report were from female dogs, two of which cultured E. coli.

Haematuria may suggest inflammation of the urinary tract and is a common clinical sign for patients with bacterial cystitis (Ettinger, 2017). Microscopic or occult haematuria may be associated with urinary tract inflammation, or may be secondary to cystocentesis collection technique (Ettinger, 2017). For these reasons we chose to include patients with occult haematuria in the analysis rather than excluding them. There was no association between patients with microscopic haematuria and positive QUC results.

In an effort to further characterise which patients may benefit most from a QUC if they have an inactive sediment examination, presenting complaints/clinical signs, pre-existing comorbidities and recent steroid and antimicrobial therapy were recorded. Previous reports have identified antimicrobial or steroid administration within 60 days to be a risk factor for a positive urine culture in dogs (Freshman et al., 1989). We did not identify an association between prior antimicrobial or steroid administration and positive QUC in this population, and the lack of association persisted when the dogs presenting for rechecks following antimicrobial therapy for bacterial cystitis were excluded from the analysis. Additionally, there was no association between specific presenting complaints/clinical signs, or pre-existing comorbidities in dogs with positive QUCs.

Three of the dogs with a positive QUC (dogs 1-3) presented to the emergency service for evaluation of simple 
and straightforward LUTS and had no other comorbidities or history of drug administration. Their histories and physical examinations were otherwise unremarkable. All three dogs were prescribed amoxicillin/clavulanic acid for a range of 7-10 days and follow-up records indicate that all dogs had resolution of their LUTS. Unfortunately, no further diagnostic evaluation beyond the inactive sediment examination and positive QUC was performed. Their sediment examination may have been inactive because it was collected too early in the course of their infection. Given their positive response to antimicrobial therapy, a sporadic bacterial cystitis is suspected. It should be noted that the International Society for Companion Animal Infectious Disease guidelines for treatment of sporadic bacterial cystitis recommend 3-5 days of antibacterial therapy. These guidelines also recommend considering a course of analgesics, such as non-steroidal anti-inflammatory drugs, and if signs persist or worsen in 3-4 days, then considering antimicrobial therapy (Weese et al., 2019).

The other three dogs with a positive QUC (dogs 4-6) presented for a variety of clinical signs with a host of preexisting comorbidities. Dog 4 was suspected of having pyelonephritis based on the presence of fever, mild aezotemia and pyelectasia with ureteral dilation, although the presence of additional disease processes could not be ruled out. Despite timely administration of antimicrobial therapy, dog 4 failed to show any clinical improvement with hospitalisation and was ultimately euthanised $<48$ hours after presentation.

Dogs 5 and 6 were suspected of having subclinical bacteriuria, which is defined as a positive QUC from a properly collected urine sample (i.e. via cystocentesis) in a patient with no LUTS (Weese et al,. 2019), although pyelonephritis could not be entirely ruled out, it was considered less likely. Dog 5 presented for haematochezia and was incidentally diagnosed with CKD during diagnostic evaluation. This is not unexpected, as it is common for dogs with CKD to have subclinical bacteriuria, although the clinical relevance of such a finding remains unknown (Foster et al., 2018). Dog 6 was a previously diagnosed diabetic who presented with ketoacidosis and was actively receiving chemotherapy for mast cell tumours. This is also unsurprising, as it is also common for dogs with DM to have subclinical bacteriuria (Forrester et al., 1999). Although both dog 5 and 6 received amoxicillin/clavulanic acid, antimicrobial treatment of subclinical bacteriuria is rarely indicated and actually discouraged in the International Society for Companion Animal Infectious Disease guidelines (Weese et al., 2019). Of course, there are exceptions and treatment for subclinical bacteriuria should be considered in patients at high risk for ascending or systemic infections, patients unable to display LUTS (i.e. those with spinal cord injury) or following the culture of plaque-forming (i.e. risk for encrusting cystitis) or urease-producing bacterial (i.e. risk for struvite uroliths formation) (Weese et al,. 2019).

This study has several important limitations. Due to the logistics of a private practice facility using an outside

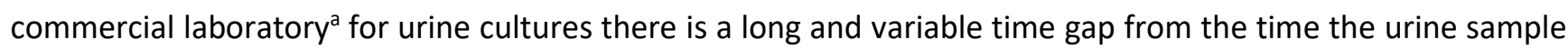
is obtained and when it is plated at the laboratory ${ }^{a}$. Weekday business hour samples, weekday after-hours samples and weekend samples were compared with no differences identified. However, it remains less than ideal to be evaluating cultures plated greater than 24 hours after collection and is an unfortunate constraint of private practice facilities that do not have on-site laboratories. This delay may have led to falsely negative results, particularly if a patient had a low number of bacteria in the sample and the sample was stored for a longer period of time. The optimal handling and storage for urine samples for which plating will be delayed is not clearly defined in veterinary medicine. Experiments by Patterson et al. (2018) showed a statistically significant decrease in E. coli CFU/mL when urine was placed in either silicone clot tubes or urine transport tubes and stored in the refrigerator for 24 hours. Conversely investigations by Acierno et al. (2018) failed to find a difference in colony counts or positive culture rate when urine was plated immediately or stored in plain glass tubes in refrigeration for 24 hours. Furthermore, Padilla et al. (1981) supports the use of refrigeration over room temperature storage for urine samples that are not plated immediately after collection with only one false negative result out of 26 QUCs and no false positive results after 24 hours of refrigeration. It is worth noting that Patterson et al. (2018) used pooled sterile urine inoculated with E. coli while Acierno et al. (2018) 
used clinical samples from dogs with a suspected bacteria cystitis. Both techniques appear throughout the literature, however we were unable to find a good description of the differences in the urinary environment between naturally and artificially infected urine, and wonder if this might contribute to the difference between these two study outcomes.

Clearly this is an area that needs further study in many regards. For example, because long delays to sample plating at commercial laboratories are the challenging reality for most private practice institutions, it would be useful to repeat the current study with a prospective design that controls time from collection to urinalysis and culture plating to determine if there is a clinically important difference in the positive culture rate. Additionally, inoculating sterile urine with a variety of different organisms at a range of different concentrations, storing that urine in the refrigerator for 24,48 and 72 hours and then plating it utilising the commercial laboratory's standard methods to determine if a positive culture rate compared to urine plated immediately after collection, would be useful information. Similarly, studies evaluating the cost and reliability of in-house urine plating, and utilisation of techniques other than urine culture to identify causative organisms and predicted antimicrobial sensitivity would be of benefit to the veterinary community.

QUCs are a relatively expensive test compared to urinalysis alone and knowing the prevalence of a positive result in a dog with inactive urine sediment can assist clinicians in evaluating the medical and financial risks vs benefits of when to order this test. Dogs displaying LUTS, even with an inactive sediment examination, may benefit from a QUC. A QUC should also be considered on a case-by-case basis for systemically ill patients with various comorbidities presenting to emergency services. Ideally urine would be cultured within 24 hours post collection, however inability to do so should not preclude culture.

Prospective studies evaluating the bacterial cystitis and subclinical bacteriuria prevalence in specific at-risk populations, studies that mimic the typical storage time and handling of urine samples submitted to commercial laboratories that are designed to determine the clinical impact of these practices, as well as research looking for rapid, point-of-care testing alternatives to urine culture and microbial sensitivity are needed in veterinary medicine.

\title{
FOOTNOTES
}

\section{Footnotes}

${ }^{a}$ Antech Diagnostics 17620 Mt. Herrmann Street, Fountain Valley, CA 92708

${ }^{b}$ Microsoft Office Excel

\author{
Abbreviations \\ $\mathrm{CFU}=$ colony forming unit \\ $C K D=$ chronic kidney disease \\ $\mathrm{DM}=$ diabetes mellitus \\ $\mathrm{HAC}=$ hyperadrenocorticism \\ LUTS = lower urinary tract signs \\ QUC = quantitative urine culture \\ USG = urine specific gravity \\ $U A=$ urinalysis
}

NFLICT OF INTEREST 
The authors declare no conflicts of interest.

\section{REFERENCES}

1. Acierno, M.J., Partyka, M., Waite, K., Cunha, A. \& Mitchell, M.A. 'Effects of refrigeration of clinical canine urine samples on quantitative bacterial culture'. Journal of the American Veterinary Medical Association (2018); 253(2): 177-180. DOI: https://doi.org/10.2460/javma.253.2.177

2. Comer, K.M. \& Ling, G.V. 'Results of urinalysis and bacterial culture of canine urine obtained by antepubic cystocentesis, catheterization and the midstream voided methods'. Journal of the American Veterinary Medical Association (1981); 179: 891-895.

3. Ettinger, S.J., Feldman, E.C. \& Côté, E. Textbook of Veterinary Internal Medicine. 8th ed. St. Louis: Elsevier, Inc. (2017).

4. Forrester, S.D., Troy, G.C., Dalton, M.N., Huffman, J.W. \& Holtzman, G. Retrospective Evaluation of Urinary Tract Infection in 42 Dogs with Hyperadrenocorticism or Diabetes Mellitus or Both'. Journal of Veterinary Internal Medicine (1999); 13: 557-560. DOI: https://doi.org/10.1111/j.19391676.1999.tb02209.x

5. Foster, J.D., Krishnan, H. \& Cole, S. 'Characterization of subclinical bacteriuria, bacterial cystitis, and pyelonephritis in dogs with chronic kidney disease'. Journal of the American Veterinary Medical Association (2018); 252(10): 1257-1262. DOI: https://doi.org/10.2460/javma.252.10.1257

6. Freshman, J.L., Reif, J.S., Allen, T.A. \& Jones, R.L. 'Risk factors associated with urinary tract infection in female dogs'. Preventive Veterinary Medicine (1989); 7(2): 59-67. DOI: https://doi.org/10.1016/0167$\underline{5877(89) 90037-8}$

7. Hall, J.L., Holmes, M.A. \& Baines, S.J. 'Prevalence and antimicrobial resistance of canine urinary tract pathogens'. Vet Record (2013); 173(22): 549. DOI: http://dx.doi.org/10.1136/vr.101482

8. Neely, J.G., Hartman, J.M., Forsen, J.W. Jr. \& Wallace, M.S. Tutorials in clinical research: VII. Understanding comparative statistics (contrast)-part B: application of T-test, Mann-Whitney U, and chi-square. Laryngoscope. (2003); 113(10):1719-1725. DOI: https://doi.org/10.1097/00005537200310000-00011

9. Padilla, J., Osborne, C.A. \& Ward, G.E. 'Effects of storage time and temperature on quantitative culture of canine urine'. Journal of the American Veterinary Medical Association (1981); 178: 1077-1081.

10. Patterson, C.A., Bishop, M.A., Pack, J.D., Cook, A.K. \& Lawhon, S.D. 'Effects of processing delay, temperature, and transport tube type on results of quantitative bacterial cultures of canine urine'. Journal of the American Veterinary Medical Association (2016); 248(2): 183-187. DOI: https://doi.org/10.2460/javma.248.2.183

11. Vetter, T.R. Fundamentals of Research Data and Variables: The Devil Is in the Details. Anesthesia \& Analgesia. (2017); 125(4):1375-1380. DOI: http://dx.doi.org/10.1213/ANE.0000000000002370

12. Weese, S.J., Blondeau, J., Boothe, D., Guardabassi, L.D., Gumley, N., Papich, M., Jessen, L.R., Lappin, M., Rankin, S., Westropp, J.L. \& Sykes, J. 'International Society for Companion Animal Infectious Diseases (ISCAID) guidelines for the diagnosis and management of bacterial urinary tract infections in dogs and cats'. The Veterinary Journal (2019); 247: 8-25.

DOI: https://doi.org/10.1016/i.tvjl.2019.02.008 


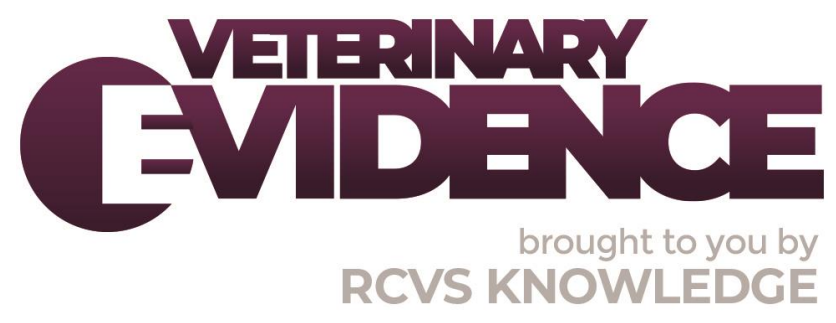

\section{Intellectual Property Rights}

Authors of Knowledge Summaries submitted to RCVS Knowledge for publication will retain copyright in their work, and will be required to grant RCVS Knowledge a non-exclusive license of the rights of copyright in the materials including but not limited to the right to publish, re-

publish, transmit, sell, distribute and otherwise use the materials in all languages and all media throughout the world, and to license or permit others to do so.

\section{Disclaimer}

Any opinions expressed in articles and other publication types published in Veterinary Evidence are the author's own and do not necessarily reflect the view of the RCVS Knowledge. Veterinary Evidence is a resource to help inform, and the content herein should not override the responsibility of the practitioner. Practitioners should also consider factors such as individual clinical expertise and judgement along with patient's circumstances and owners' values. Authors are responsible for the accuracy of the content. While the Editor and Publisher believe that all content herein are in accord with current recommendations and practice at the time of publication, they accept no legal responsibility for any errors or omissions, and make no warranty, express or implied, with respect to material contained within.

For further information please refer to our Terms of Use.

RCVS Knowledge is the independent charity associated with the Royal College of Veterinary Surgeons (RCVS). Our ambition is to become a global intermediary for evidence based veterinary knowledge by providing access to information that is of immediate value to practicing veterinary professionals and directly contributes to evidence based clinical decision-making.

\section{https://www.veterinaryevidence.org/}

RCVS Knowledge is a registered Charity No. 230886.

Registered as a Company limited by guarantee in England and Wales No. 598443.

Registered Office: Belgravia House, 62-64 Horseferry Road, London SW1P 2AF

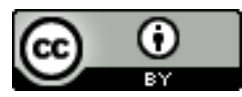

This work is licensed under a Creative Commons Attribution 4.0 International License. 